\title{
Prediction of successful caudal epidural injection using color Doppler ultrasonography in the paramedian sagittal oblique view of the lumbosacral spine
}

\author{
Seon Woo Yoo ${ }^{1,2,3}$, Min-Jong $\mathrm{Ki}^{1,2,3}$, A Ram Doo ${ }^{1,2,3}$, Cheol Jong Woo ${ }^{1}$, Ye Sull Kim ${ }^{1}$, and Ji-Seon Son ${ }^{1,2,3}$ \\ 'Department of Anesthesiology and Pain Medicine, Jeonbuk National University Medical School and Hospital, Jeonju, Korea \\ ${ }^{2}$ Research Institute of Clinical Medicine of Jeonbuk National University, Jeonju, Korea \\ ${ }^{3}$ Biomedical Research Institute of Jeonbuk National University Hospital, Jeonju, Korea
}

Received February 24, 2021

Revised May 25, 2021

Accepted May 26, 2021

Handling Editor: Young Hoon Kim

\section{Correspondence}

Ji-Seon Son

Department of Anesthesiology and Pain Medicine, Jeonbuk National University Medical School and Hospital, Research Institue of Clinical Medicine of Jeonbuk National University, 20, Geonji-ro, Deokjin-gu, Jeonju 54907, Korea Tel: +82-63-250-1241

Fax: +82-63-250-1240

E-mail: sjs6803@jbnu.ac.kr
Background: Ultrasound-guided caudal epidural injection (CEI) is limited in that it cannot confirm drug distribution at the target site without fluoroscopy. We hypothesized that visualization of solution flow through the inter-laminar space of the lumbosacral spine using color Doppler ultrasound alone would allow for confirmation of drug distribution. Therefore, we aimed to prospectively evaluate the usefulness of this method by comparing the color Doppler image in the paramedian sagittal oblique view of the lumbosacral spine (LS-PSOV) with the distribution of the contrast medium observed during fluoroscopy.

Methods: Sixty-five patients received a 10-mL CEI of solution containing contrast medium under ultrasound guidance. During injection, flow was observed in the LSPSOV using color Doppler ultrasonography, following which it was confirmed using fluoroscopy. The presence of contrast image at L5-S1 on fluoroscopy was defined as "successful CEI." We then calculated prediction accuracy for successful CEI using color Doppler ultrasonography in the LS-PSOV. We also investigated the correlation between the distribution levels measured via color Doppler and fluoroscopy. Results: Prediction accuracy with color Doppler ultrasonography was $96.9 \%$. The sensitivity, specificity, positive predictive value, and negative predictive value were $96.7 \%, 100 \%, 100 \%$, and $60.0 \%$, respectively. In 52 of 65 patients (80\%), the highest level at which contrast image was observed was the same for both color Doppler ultrasonography and fluoroscopy.

Conclusions: Our findings demonstrate that color Doppler ultrasonography in the LS-PSOV is a new method for determining whether a drug solution reaches the lumbosacral region (i.e., the main target level) without the need for fluoroscopy.

Key Words: Anesthesia, Caudal; Contrast Media; Equivalence Trial; Fluoroscopy; Injections, Epidural; Low Back Pain; Lumbar Vertebrae; Sensitivity and Specificity; Ultrasonography, Doppler, Color; Ultrasonography, Interventional.

\section{INTRODUCTION}

Lumbosacral epidural injection is commonly performed in patients with lower back pain or radiating pain in the lower extremities. The long-term effect of lumbosacral epidural injection remains controversial, but many stud- (a) This is an open-access article distributed under the terms of the Creative Commons Attribution Non-Commercial License (http://creativecommons.org/licenses/by-nc/4.0/), which permits unrestricted non-commercial use, distribution, and reproduction in any medium, provided the original work is properly cited.

(C) The Korean Pain Society, 2021
Author contributions: Seon Woo Yoo: Writing/manuscript preparation; Min-Jong Ki: Investigation; A Ram Doo: Investigation; Cheol Jong Woo: Data curation; Ye Sull Kim: Investigation; Ji-Seon Son: Study conception. 
ies have shown a favorable trend regarding various effects including short-term benefits [1-4]. Among the several epidural approaches, caudal epidural injection (CEI) is preferred by many clinicians because it can be implemented easily. CEI is possible using a blind technique, which is less invasive and requires a short procedure time. However, the failure rate of blind techniques in adults is as high as $25 \%$, even for experienced clinicians, because of variations in caudal anatomy [5-7].

Recently, ultrasound guidance has increased the success rate of CEI, with several studies showing that ultrasound is effective in guiding needle placement during CEI $[8,9]$. Ultrasound can be used to accurately assess caudal anatomical structures such as the sacral hiatus and sacral cornu before inserting the needle and to evaluate needle placement in real time $[10,11]$. Nevertheless, fluoroscopyguided caudal epidural injection (FG-CEI) is still considered the gold standard, given the need to confirm the distribution of the solution in the epidural space $[5,7,12,13]$. It has been considered impossible to confirm the distribution of the injected solution at the lumbosacral level when using ultrasound-guided CEI (USG-CEI), although it is effective in guiding needle placement.

Compared to FG-CEI, USG-CEI has advantages such as ease of use, short procedural time, and contrast-free and radiation-free application. In rare cases, however, USGCEI may result in the injected solution not reaching the desired target level, which is usually the lumbosacral epidural space. When performing a CEI with ultrasound alone, it is difficult to predict the area over which the injected solution has spread. Therefore, CEI-which is com- monly recommended in clinical practice-is performed as follows. First, the sacral area is scanned using ultrasound, the needle entry point is checked, and the needle is inserted. After needle placement, fluoroscopy is used to examine the distribution of the contrast medium, following which the drug solution is injected. The identification of contrast medium via fluoroscopy is considered essential for predicting whether a drug has reached the target site [14].

The paramedian sagittal oblique view of the lumbosacral spine (LS-PSOV) is one of the ultrasonographic views introduced for neuraxial anesthesia $[15,16]$. In the LSPSOV, one can observe the laminae of each lumbar spine, as well as the anterior and posterior complexes containing the dura mata (Fig. 1). Therefore, we speculated that the use of color Doppler ultrasonography would allow us to confirm the distribution of the injected solution in the epidural space at the lumbosacral level without the need for fluoroscopy. In other words, we hypothesized that color Doppler flow in the LS-PSOV during ultrasound could replace identification of contrast medium via fluoroscopy. Thus, the present study aimed to evaluate the validity and reliability of CEI using color Doppler ultrasonography in the LS-PSOV by comparing the technique with fluoroscopy.
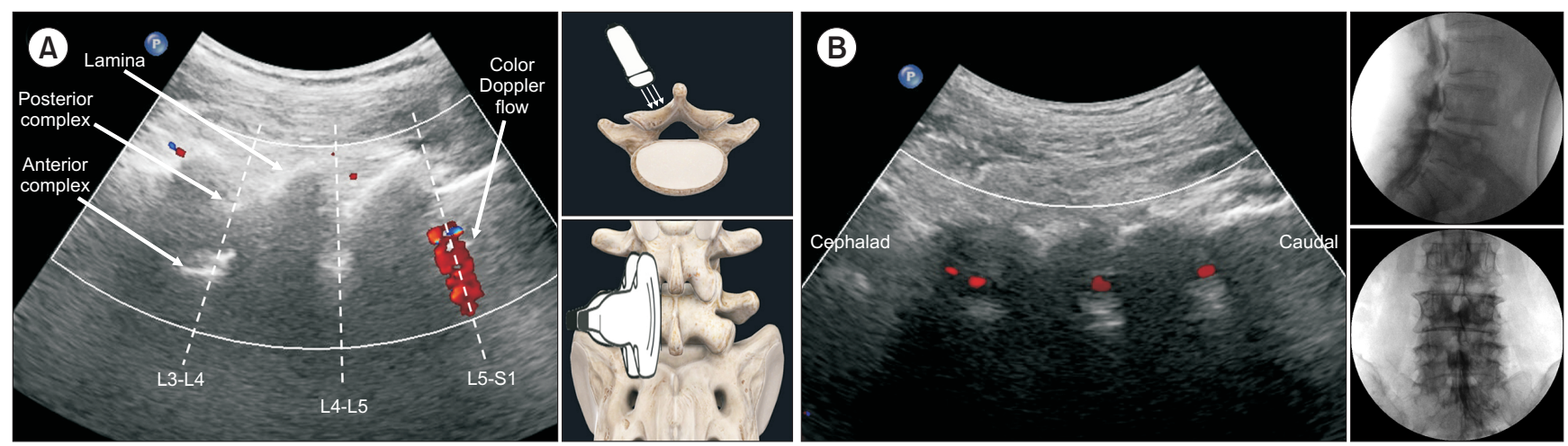

Fig. 1. Ultrasound paramedian sagittal oblique view (PSOV) of the lumbosacral level (A). The patient was in the prone position, and the convex ultrasound probe was placed on the patient's back aligned to the sagittal plane (median sagittal view). The sacrum can be recognized as a horizontal high-echo curve structure, and the L5 layer has a typical "serrated" shape. After checking the sacrum, the sacrum is placed on the right side of the ultrasound window through the cephalad shift of the probe, and the L3 spinous process is visible on the left side. The probe was then shifted by 1-2 fingers in the left lateral direction to ensure that the lamina was visible (paramedian sagittal view). The probe was then tilted slightly toward the midline of the spine (PSOV). In the image presented here, a serrated laminar shape is visible, and hyperechoic shadows of the anterior complex and posterior complex including the dura mater can be observed between the lamina of each level. The color Doppler in (A) shows that the injection flow has reached L5-S1. (B) is a color Doppler image of another patient performed in the same way. The drug solution rises from the caudal region to the cephalad, and Doppler flow can be observed between each lamina. Doppler flow can be observed at L5-S1, L4-L5, and L3-L4, and the anteroposterior and lateral fluoroscopy images show that the contrast medium is distributed at the corresponding levels. 


\section{MATERIALS AND METHODS}

\section{Participants}

This prospective study was approved by the Institutional Review Board of Jeonbuk National University Hospital (No. CUH 2019-08-058) and was registered with the Clinical Research Information Service (CRIS) (No. KCT0005682). We obtained written informed consent from all patients, and the research was performed in accordance with the Ethical Principles for Medical Research Involving Human Subjects, outlined in the World Medical Association's Declaration of Helsinki. The study included 65 adult patients in our pain clinic, aged $19-80$ years, who required CEI for low back pain with or without radiculopathy. Patients with a history of lumbar spine surgery, allergies to local anesthetics or contrast medium, coagulation abnormalities, suspected infections in the coccygeal area, and pregnant women were excluded from the study. The flow diagram of patient selection is presented in Fig. 2.

\section{Procedure}

Patients were in the prone position, and a pillow was placed under the lower abdomen to expose the insertion site. The area around the coccyx was thoroughly sterilized with $10 \%$ povidone iodine. We decided to inject a 10 $\mathrm{mL}$ dose based on a pilot study targeting the lower lumbar level (i.e., L3/4 or L4/5). A 10-mL syringe connected to a 25 -gauge, $4-\mathrm{cm}$-long needle and a 30 -cm-long extension line was prepared. The $10-\mathrm{mL}$ drug solution contained 4 $\mathrm{mL}$ of contrast medium (Iohexol, $300 \mathrm{mg} \mathrm{I} / \mathrm{mL}$, Omnipaque $^{\mathrm{TM}}$; GE Healthcare, Shanghai, China), $0.5 \mathrm{~mL}$ of dexamethasone $(5 \mathrm{mg} / \mathrm{mL} / \mathrm{amp}), 2.5 \mathrm{~mL}$ of lidocaine $(20 \mathrm{mg} /$ $\mathrm{mL}$ ), and $3 \mathrm{~mL}$ of normal saline. After checking the sacral hiatus and sacrococcygeal ligament using a 4-18 $\mathrm{MHz}$ linear probe (Affiniti 70; Philips Ultrasound, Cambridge, $\mathrm{MA})$, the needle entry point was confirmed, and the needle was inserted toward the target point in real time. We then used a 2-6 MHz convex probe (Affiniti 70; Philips Ultrasound) to observe the Doppler flow in the LS-PSOV [16]. The operator fixed the convex probe in the LS-PSOV firmly with the left hand (Fig. 1) and carefully observed whether the Doppler image appeared while slowly injecting the drug solution with the right hand after confirming negative blood aspiration. To discriminate against the noise signal of the ultrasonography, the same person held the probe and injected the drug solution. During injection of the drug solution, the operator evaluated the level at which the color Doppler image was visible. After injecting all 10 $\mathrm{mL}$, anterior-posterior and lateral fluoroscopic images were documented and saved for use by another physician who would later assess the image blindly. The highest level observed by the color Doppler ultrasonography and fluoroscopy was classified as "not detected," "L5-S1," "L4-L5," or "L3-L4" (Fig. 1). Recorded events included unexpected adverse reactions or unintended injections (e.g., intravascular and intrathecal).

\section{Data analysis and statistical analysis}

The observation of contrast image observed at L5-S1 on fluoroscopy was defined as "successful CEI." The primary outcome was the probability of predicting successful CEI using color Doppler ultrasonography in LS-PSOV, defined as "prediction accuracy" and calculated as follows: $(A+$ $B)$ / Total number of patients analyzed. In this equation, $A$ refers to patients in whom both Doppler flow of ultrasonography and the contrast image of fluoroscopy allowed for observation in the LS-PSOV and at L5-S1, respectively. $B$ refers to patients in whom both Doppler flow and the contrast image did not allow for observation in both the LS-PSOV and at L5-S1, respectively. We also calculated the sensitivity, specificity, positive predictive value, and negative predictive value to verify the CEI using color Doppler ultrasonography in the LS-PSOV when compared with the FG-CEI, which is known as the gold standard. Fig. 3 shows how the levels of injected drug distribution predicted by

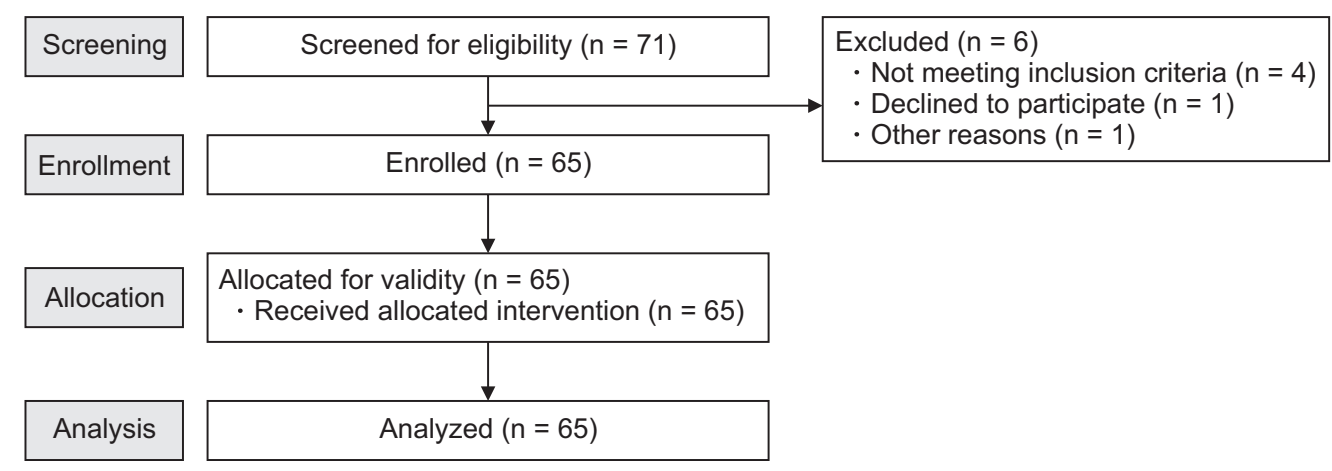

Fig. 2. Consolidated Standards of Reporting Trials (CONSORT) flow diagram. 


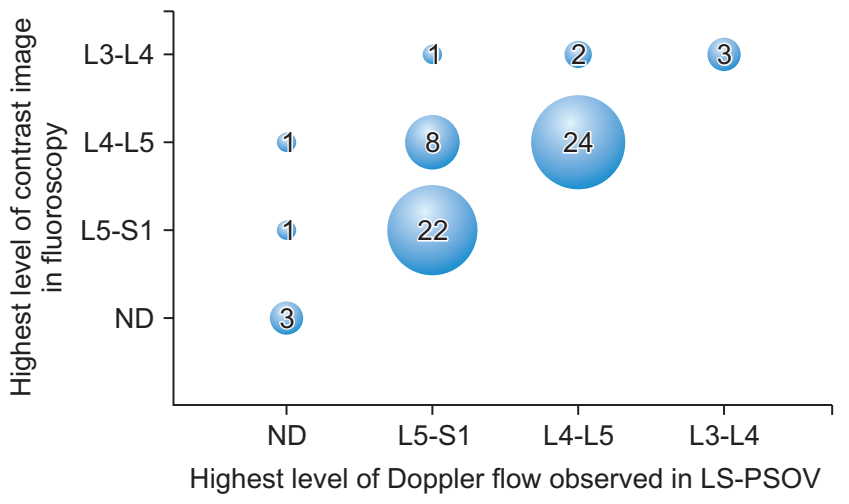

Fig. 3. The figure shows the correlation between the highest level of injection flow measured using color Doppler ultrasonography in the paramedian sagittal oblique view at the lumbosacral level (LS-PSOV) and the highest level at which contrast image was identified using fluoroscopy. In 52 of 65 patients $(80 \%)$, the highest level at which image (Doppler flow or contrast medium) was observed was the same for both color Doppler ultrasonography and fluoroscopy: not detected $(n=3)$, L5-S1 $(n=22)$, L4-L5 $(n=24)$, L3-L4 $(n=3)$. The difference in the level measured by the two modalities was one level in 11 patients and two levels in two patients (16.9\% and 3.0\%, respectively). ND: not detected.

Table 1. Patient characteristics

\begin{tabular}{lc}
\hline \multicolumn{1}{c}{ Characteristic } & Patients $(\mathrm{n}=65)$ \\
\hline Sex (male:female) & $32: 33$ \\
Age $(\mathrm{yr})$ & $61(46-69)$ \\
Height $(\mathrm{cm})$ & $162.0(154.5-170.0)$ \\
Weight $(\mathrm{kg})$ & $62(53-70)$ \\
Pre-CEl pain score (VAS) & $6.5 \pm 2.2$ \\
Post-CEl pain score (VAS) & $3.5 \pm 2.2$ \\
\hline
\end{tabular}

Values are expressed as numbers, medians (25th-75th percentile), or means \pm standard deviation.

CEl: caudal epidural injection, VAS: visual analogue scale.

color Doppler ultrasonography match those identified by fluoroscopy.

We performed a non-inferiority test (one-sided test) of CEI using color Doppler in the LS-PSOV for FG-CEI and set $-10 \%$ as a non-inferiority margin based on similar previous studies $[17,18]$. The sample size was determined based on the expected prediction accuracy of CEI using Doppler ultrasonography in the LS-PSOV. We expected the probability of detecting the injected solution flow passing through L5-S1 to be approximately $80 \%$ based on our pilot study and our own retrospective data. With a type 1 error of 0.05 , power of $80 \%$, discordant ratio of 0.15 , and drop out of 0.15 , the minimal sample size was estimated to be 65 . Statistical analysis was performed using PASW Statistics 18.0 (IBM Co., Armonk, NY).
Table 2. The contrast image in fluoroscopy at L5-S1 after CEI using color Doppler ultrasound in LS-PSOV

\begin{tabular}{ccc}
\hline Doppler flow of & \multicolumn{2}{c}{ The contrast image of fluoroscopy at L5-S1 } \\
\cline { 2 - 3 } $\begin{array}{c}\text { ultrasonography in the } \\
\text { LS-PSOV }\end{array}$ & Positive & Negative \\
\hline Positive & 60 & 0 \\
Negative & 2 & 3 \\
\hline
\end{tabular}

CEl: caudal epidural injection, LS-PSOV: the paramedian sagittal oblique view at the lumbosacral level.

Table 3. Prediction accuracy of USG-CEI vs. FG-CEI $(n=65)$

\begin{tabular}{lll}
\hline \multicolumn{1}{c}{ Result } & \multicolumn{1}{c}{ USG-CEI } & \multicolumn{1}{c}{ FG-CEI } \\
\hline Prediction accuracy & $63 / 65(96.9 \%)$ & $65 / 65(100 \%)$ \\
Absolute difference vs. FG-CEI & $-3.1 \%(-7.4 \%$ to 1.2\%) & \\
(95\% Cl) & & \\
\hline
\end{tabular}

USG-CEl: ultrasound-guided caudal epidural injection, FG-CEl: fluoroscopy-guided caudal epidural injection, Cl: confidence interval.

\section{RESULTS}

Sixty-five patients were enrolled, and all 65 patients were analyzed without exclusion. The baseline characteristics of the patients are described in Table 1 . The mean patient age was 61 years, the pre-CEI visual analogue scale (VAS) score was 6.5 , and the post-CEI VAS score was $3.5(P<$ $0.001)$.

In 62 of 65 patients, the contrast image was observed in L5/S1 on fluoroscopy after USG-CEI, and the probability of "successful CEI" was 95.4\% (Tables 2, 3). The prediction accuracy of CEI using Doppler ultrasonography in the LS-PSOV, the primary outcome of this study, was $96.9 \%$. That is, in all 60 patients with Doppler flow observed in the LS-PSOV, contrast images were also observed at L5-S1 on fluoroscopy, and in all three patients without Doppler flow, no contrast images were observed. Two of 65 patients exhibited no Doppler flow, although contrast images were observed at L5-S1 on fluoroscopy; consequently, the prediction of color Doppler ultrasonography was incorrect. On the other hand, none of the patients showed both positive Doppler flow and negative contrast image. The sensitivity, specificity, positive predictive value, and negative predictive value of color Doppler ultrasonography in the LS-PSOV were $96.7 \%, 100 \%, 100 \%$, and $60.0 \%$, respectively. In addition, Fig. 3 shows how the level of injected drug distribution predicted by color Doppler ultrasonography match that confirmed by fluoroscopy.

In the non-inferiority test, CEI performed under Doppler ultrasonography in the LS-PSOV was not inferior to that performed under FG, since the lower limit of the confidence interval for the absolute difference versus FG-CEI 
was greater than the pre-specified non-inferiority margin of $-10 \%$. Among the 65 patients, there were no unexpected adverse reactions or unintended injections (e.g., intrathecal or intravascular).

\section{DISCUSSION}

Fluoroscopy is currently the standard for predicting drug distribution in the epidural space before drug injection. Although ultrasound has many advantages over fluoroscopy and is widely used, the visualization of solution spread has only been possible using fluoroscopy [19]. In the present study, we investigated whether ultrasound could confirm drug spread during CEI. Ultrasound has many advantages over fluoroscopy for CEI: it is radiationfree, contrast-free, and easy to use and requires a short procedure time [9]. Above all, USG-CEI provides a more accurate route for needle insertion than FG-CEI because it can provide information regarding anatomical structures such as the sacral hiatus, sacral cornu, and sacrococcygeal ligament in real time [20].

Our study introduced and verified a novel ultrasonic method for predicting the range of drug spread and confirming the success of CEI, with a very high prediction accuracy of $96.9 \%$. Among the 65 patients, failure to predict the success of CEI occurred in only two patients. The statistical significance obtained from prediction accuracy was also not inferior to that of FG-CEI. In particular, the $100 \%$ specificity is very impressive. This means that the probability of a type 1 error is almost zero. In addition, if color Doppler flow is observed at L5-S1, the probability that the actual drug solution reaches above L5-S1 is $100 \%$. If it is observed at L4-L5, the probability that the drug solution reaches above L4-L5 is also $100 \%$.

Failure to predict successful epidural spread of the injected solution occurred in two patients. In these two patients, injection flow was not observed on color Doppler ultrasonography, but the epidural spread of the contrast solution was observed at L5-S1 under fluoroscopy. One patient had morbid obesity (body mass index: 35.2), and the other patient exhibited severe degenerative changes. These factors are thought to have influenced color Doppler detection $[21,22]$. One of the limitations of ultrasound is that it remains difficult to check for unintended intravascular injections and dura puncture [5]. However, injecting immediately after penetrating the sacrococcygeal ligament is associated with a very low risk of intravascular injection or dura puncture [23]. In addition, a previous study identified that intravascular injection can be distinguished even by color Doppler ultrasound flow in the longitudinal view of the sacral hiatus [11]. In our study, none of the 65 patients received any unintended injection (e.g., intrathecal or intravascular).

Our findings indicated that color Doppler ultrasonography in the LS-PSOV can predict the level to which the injected solution has spread in real time and in $80 \%$ of patients. Indeed, the level was exactly consistent with the fluoroscopic confirmation in 52 of 65 patients (Fig. 3). These findings suggest that it is possible to determine whether the injected solution has reached the target level in real time, and that this method can help determine the volume of solution injected, which may in turn prevent overuse of the injected drug. When Doppler artifacts such as noise or blood vessels are suspected, the operator can distinguish them from the actual solution flow by repeatedly injecting and stopping. As in our study, this is simplified when a single operator holds the probe and injects the solution simultaneously. For consistency, all procedures were performed in the left PSOV in the present study. However, we recommend selecting the left or right PSOV based on the desired target side.

This study has several limitations. First, we did not examine solution spread above level L2-L3 on ultrasound. Since issues at L2-L3 or higher are less likely to result in lower back pain and the distance from the CEI insertion site is far, there are relatively few cases in which CEI is indicated at L2-L3. In addition, we were unable to capture L5-S1 through L2-L3 on a single ultrasound screen, making it difficult to evaluate the spread of contrast solution in these regions at the same time. Even within one ultrasound window, as the level of the lumbar spine increased, it became more difficult to observe the color Doppler flow. In particular, the amount of color Doppler flow was quite small at L3-L4, necessitating more careful examination. A larger solution volume may have allowed for easier visualization of Doppler flow. However, we thought that high doses of lidocaine pose a risk of local anesthetic systemic toxicity because ultrasound cannot complet rule out intravascular injection [24]. In addition, according to our pilot study and our own retrospective data, even the $10 \mathrm{~mL}$ dose reached the level of L4-5 or L5-S1 in most patients, and it was thought that an excessive dose could rather obscure the comparison between the ultrasound and fluoroscopy in the lower lumbar level, one of the objectives of this study.

Second, when there is vascular uptake during the epidurogram, it cannot be excluded by ultrasound alone. This is thought to be a phenomenon caused by the vein in the epidural space and is distinguished from vascular injection. In our study, several vascular uptakes were identified on fluoroscopy during the epidurogram, but there was no local anesthetic systemic toxicity, including neurological symptoms. Third, the possibility that the vertebra, which 
is expected to be L5 on ultrasound, is actually S1, due to lumbarization of S1, cannot be excluded. Although accurate lumbar vertebra numbering is possible through whole spine images [25], preoperative whole spine images of all patients were not obtained uniformly.

Finally, color Doppler flow was not easily observed in patients with narrow interlaminar spaces or severe scoliosis due to advanced degenerative changes. Furthermore, the ultrasound window was obscured depending on the degree of obesity, further complicating evaluation. Further studies are required to determine whether color Doppler ultrasonography in the LS-PSOV can be used to evaluate CEI of contrast material in various patient groups.

In conclusion, our findings suggest that CEI using color Doppler ultrasonography in the LS-PSOV has good validity, reliability, and feasibility. Our findings indicated that this technique was associated with high prediction accuracy and was not inferior to FG-CEI. Moreover, our study suggests that color Doppler ultrasonography in the LS-PSOV is easy to apply in clinical practice, allowing for visual confirmation that the CEI solution has reached the target level of the lumbosacral spine without the need for fluoroscopy.

\section{CONFLICT OF INTEREST}

No potential conflict of interest relevant to this article was reported.

\section{FUNDING}

This paper was supported by Fund of Biomedical Research Institute, Jeonbuk National University Hospital.

\section{ORCID}

Seon Woo Yoo, https://orcid.org/0000-0002-1742-7487

Min-Jong Ki, https://orcid.org/0000-0001-9959-7908

A Ram Doo, https://orcid.org/0000-0003-1310-790X

Cheol Jong Woo, https://orcid.org/0000-0003-4550-4336

Ye Sull Kim, https://orcid.org/0000-0001-8771-488X

Ji-Seon Son, https://orcid.org/0000-0002-6672-4576

\section{REFERENCES}

1. Oliveira CB, Maher CG, Ferreira ML, Hancock MJ, Oliveira VC, McLachlan AJ, et al. Epidural corticosteroid injections for lumbosacral radicular pain. Cochrane Database Syst Rev
2020; 4: CD013577.

2. Oliveira CB, Maher CG, Ferreira ML, Hancock MJ, Oliveira VC, McLachlan AJ, et al. Epidural corticosteroid injections for sciatica: an abridged Cochrane systematic review and meta-analysis. Spine (Phila Pa 1976) 2020; 45: E1405-15.

3. Yang S, Kim W, Kong HH, Do KH, Choi KH. Epidural steroid injection versus conservative treatment for patients with lumbosacral radicular pain: a meta-analysis of randomized controlled trials. Medicine (Baltimore) 2020; 99: e21283.

4. McLain RF, Kapural L, Mekhail NA. Epidural steroid therapy for back and leg pain: mechanisms of action and efficacy. Spine J 2005; 5: 191-201.

5. Stitz MY, Sommer HM. Accuracy of blind versus fluoroscopically guided caudal epidural injection. Spine (Phila Pa 1976) 1999; 24: 1371-6.

6. Barham G, Hilton A. Caudal epidurals: the accuracy of blind needle placement and the value of a confirmatory epidurogram. Eur Spine J 2010; 19: 1479-83.

7. el-Khoury GY, Ehara S, Weinstein JN, Montgomery WJ, Kathol MH. Epidural steroid injection: a procedure ideally performed with fluoroscopic control. Radiology 1988; 168: 554-7.

8. Nikooseresht M, Hashemi M, Mohajerani SA, Shahandeh F, Agah M. Ultrasound as a screening tool for performing caudal epidural injections. Iran J Radiol 2014; 11: e13262.

9. Chen CP, Tang SF, Hsu TC, Tsai WC, Liu HP, Chen MJ, et al. Ultrasound guidance in caudal epidural needle placement. Anesthesiology 2004; 101: 181-4.

10. Kim YH, Park HJ, Cho S, Moon DE. Assessment of factors affecting the difficulty of caudal epidural injections in adults using ultrasound. Pain Res Manag 2014; 19: 275-9.

11. Yoon JS, Sim KH, Kim SJ, Kim WS, Koh SB, Kim BJ. The feasibility of color Doppler ultrasonography for caudal epidural steroid injection. Pain 2005; 118: 210-4.

12. Renfrew DL, Moore TE, Kathol MH, el-Khoury GY, Lemke JH, Walker CW. Correct placement of epidural steroid injections: fluoroscopic guidance and contrast administration. AJNR Am J Neuroradiol 1991; 12: 1003-7.

13. Manchikanti L, Cash KA, Pampati V, McManus CD, Damron KS. Evaluation of fluoroscopically guided caudal epidural injections. Pain Physician 2004; 7: 81-92.

14. Huang J. Disadvantages of ultrasound guidance in caudal epidural needle placement. Anesthesiology 2005; 102: 693.

15. Yoo S, Kim Y, Park SK, Ji SH, Kim JT. Ultrasonography for lumbar neuraxial block. Anesth Pain Med (Seoul) 2020; 15: 397-408.

16. Chin KJ, Karmakar MK, Peng P. Ultrasonography of the adult thoracic and lumbar spine for central neuraxial blockade. Anesthesiology 2011; 114: 1459-85.

17. Van Besien J, Uvin P, Hermie I, Tailly T, Merckx L. Ultrasonography is not inferior to fluoroscopy to guide extracorporeal shock waves during treatment of renal and upper ure- 
teric calculi: a randomized prospective study. Biomed Res Int 2017; 2017: 7802672.

18. El-Yahchouchi C, Geske JR, Carter RE, Diehn FE, Wald JT, Murthy NS, et al. The noninferiority of the nonparticulate steroid dexamethasone vs the particulate steroids betamethasone and triamcinolone in lumbar transforaminal epidural steroid injections. Pain Med 2013; 14: 1650-7.

19. Yokoyama M, Hanazaki M, Fujii H, Mizobuchi S, Nakatsuka $\mathrm{H}$, Takahashi $\mathrm{T}$, et al. Correlation between the distribution of contrast medium and the extent of blockade during epidural anesthesia. Anesthesiology 2004; 100: 1504-10.

20. Senkal S, Sir E. Comparison of ultrasonography and conventional fluoroscopy guided caudal epidural injection in chronic low back pain. Turk Neurosurg 2021; 31: 119-23.

21. Dashe JS, McIntire DD, Twickler DM. Effect of maternal obesity on the ultrasound detection of anomalous fetuses.
Obstet Gynecol 2009; 113: 1001-7.

22. Modica MJ, Kanal KM, Gunn ML. The obese emergency patient: imaging challenges and solutions. Radiographics 2011; 31: 811-23.

23. Doo AR, Kim JW, Lee JH, Han YJ, Son JS. A comparison of two techniques for ultrasound-guided caudal injection: the influence of the depth of the inserted needle on caudal block. Korean J Pain 2015; 28: 122-8.

24. El-Boghdadly K, Pawa A, Chin KJ. Local anesthetic systemic toxicity: current perspectives. Local Reg Anesth 2018; 11: 3544.

25. Doo AR, Lee J, Yeo GE, Lee KH, Kim YS, Mun JH, et al. The prevalence and clinical significance of transitional vertebrae: a radiologic investigation using whole spine spiral three-dimensional computed tomographic images. Anesth Pain Med (Seoul) 2020; 15: 103-10. 